\title{
Essais
}

ESSAIS

Revue interdisciplinaire d'Humanités

Narration et lien social

\section{La mise en récit des dissidences religieuses}

Quelques réflexions à propos des hérésies médiévales en forme de bilan critique

\section{Matthieu Montantou}

\section{(2) OpenEdition}

1 Journals

Édition électronique

URL : https://journals.openedition.org/essais/9345

DOI : 10.4000/essais. 9345

ISSN : 2276-0970

Éditeur

École doctorale Montaigne Humanités

Édition imprimée

Date de publication : 15 mai 2013

Pagination : 121-138

ISBN : 978-2-9544269-0-7

ISSN : 2417-4211

Référence électronique

Matthieu Montantou, «La mise en récit des dissidences religieuses », Essais [En ligne], 3 | 2013, mis en ligne le 01 septembre 2021, consulté le 08 septembre 2021. URL : http://journals.openedition.org/ essais/9345; DOI : https://doi.org/10.4000/essais.9345 


\title{
La mise en récit des dissidences religieuses \\ Quelques réflexions à propos des hérésies médiévales en forme de bilan critique
}

\author{
Matthieu Montantou
}

Il faut le dire d'emblée : l'enjeu des lignes qui vont suivre est des plus restreints. Pas d'artifice protocolaire dans cette proclamation d'humilité, puisqu'il ne s'agit ici que de formuler quelques remarques à propos des hérésies médiévales. Leur présence dans ce recueil tient au fait qu'il me semble que les formes marginales et dissidentes de spiritualité au Moyen Âge offrent à l'étude des processus de mise en récit du lien social un terrain d'étude particulièrement riche et stimulant.

\section{Contre les discours, tout contre}

Dans une œuvre fameuse, maintes fois citée et commentée, bien plus sans doute qu'elle ne fut lue, Jean-François Lyotard concluait une enquête sur la construction des savoirs au $\mathrm{XX}^{\mathrm{e}}$ siècle en diagnostiquant la fin de la modernité et l'entrée dans la condition postmoderne de la production de la connaissance : le symptôme de ce basculement était à situer selon lui dans la mise à mal des

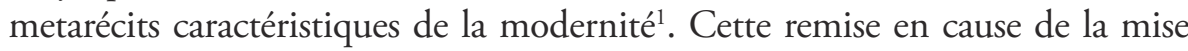
en récit a très vite été exportée au-delà de la simple analyse du champ de la connaissance contemporaine. De ce point de vue, on peut remarquer qu'il existe une tendance très nette de la recherche historique française, au moins depuis les années 1970, à déconstruire les représentations convenues et les rouages de leur constitution ; ainsi certains travaux phares de l'école historique française doivent beaucoup au concept de « discours " hérité de Michel Foucault, qui l'a placé au cœur de son projet d'archéologie des savoirs et qu'il situe justement entre les mots et les choses ${ }^{2}$. Il est d'ailleurs frappant de noter que la recherche

1 Jean-François Lyotard, La Condition postmoderne. Rapport sur le savoir, Paris, Éditions de Minuit, 1979.

2 Michel Foucault, "Entretien avec Jean-Jacques Brochier ", dans Dits et Écrits I. 1954-1975, Paris, Gallimard, coll. "Quarto », p. 804 : « [j’ai voulu] analyser les discours eux-mêmes, c'est-àdire ces pratiques discursives qui sont intermédiaires entre les mots et les choses. Ces pratiques discursives à partir desquelles on peut définir ce que sont les choses et repérer l'usage des 
actuelle s'inspire assez largement de ces grilles de lecture : à preuve, la récente parution d'un recueil d'études réunies sous un titre qui reprend un programme de recherche exploré par Foucault, la "fabrique des normes " ${ }^{3}$. À preuve aussi, les développements riches et féconds qui touchent aujourd'hui l'historiographie des formes dissidentes de croyance religieuse au Moyen Âge.

De ce point de vue, l'historien, ou l'anthropologue, des hérésies médiévales est aujourd'hui confronté à une double problématique. Il lui faut, tout d'abord, débusquer la constitution des discours, qui irriguent profondément les représentations et les imaginaires, les pratiques et les institutions. Autrement dit, un premier ensemble du questionnement touche à la façon dont les communautés religieuses dissidentes sont mises en mot par ceux qui en ont laissé une trace, une "archive $»^{4}$ : mises en mot, c'est-à-dire mises en forme et mises en scène. Les discours agissent, et ce d'autant plus que les documents qui donnent à voir les communautés dissidentes sont très majoritairement des œuvres de combat, issues de l'orthodoxie militante, et posent à ce titre plus de problèmes qu'ils n'apportent de réponse. Le premier travail consiste donc à extraire la réalité sociale des discours dans lesquels elle est enveloppée, et à en montrer la constitution : à retrouver, derrière la narration, l'authenticité d'un lien social.

La seconde étape du travail concerne plus directement le chercheur - et c'est en cela que ces remarques intéressent, au-delà des seuls médiévistes, tout chercheur en sciences humaines. Comment, en effet, ne pas ajouter un récit au récit, ou plutôt comment ne pas construire un récit en lieu et place de celui que l'on s'est attaché à déconstruire et dont on révèle les rouages ? C'est la question centrale que pose la crise postmoderne diagnostiquée par Lyotard, l'urgence à laquelle elle impose de répondre : retrouver des critères légitimant le discours du scientifique.

Ces deux problématiques semblent distinctes : elles sont au contraire étroitement liées. Elles questionnent toutes les deux la capacité du chercheur, dont l'ambition est de produire un savoir scientifique, à gérer les processus de mise en récit, soit de l'extérieur, en tant qu'observateur critique et attentif, soit

mots ». Il n'est pas question ici de comparer ce qui rapproche et ce qui distingue les " métarécits " dont parle Lyotard et les " discours " de Foucault : la question est passionnante mais elle excède les limites du présent article. Il s'agit seulement pour moi de souligner que leur introduction dans le champ de l'épistémologie est concomitante, et que les deux notions ont participé à forger des grilles conceptuelles et méthodologiques fécondes en histoire, et notamment pour l'histoire des hérésies médiévales.

3 Véronique Beaulande-Barraud, Julie Claustre, Elsa Marmursztejn (dir.), La Fabrique de la norme. Lieux et modes de production des normes au Moyen Âge et à l'époque moderne, Rennes, Presses Universitaires de Rennes, 2012.

4 Michel Foucault, ibid., p. 800 : «J'entends par archive l'ensemble des discours effectivement prononcés ; et cet ensemble de discours est envisagé non pas seulement comme un ensemble d'événements qui auraient eu lieu une fois pour toutes et qui resteraient en suspens, dans les limbes ou dans le purgatoire de l'histoire, mais aussi comme un ensemble qui continue à fonctionner, à se transformer à travers l'histoire, à donner possibilité d'apparaître à d'autres discours ". 
de l'intérieur : méfiant à l'égard des metarécits et des discours, le chercheur est pourtant au cœur des rouages de leur constitution. Ce sont ces deux aspects ambivalents, ambigus et complexes de la position du chercheur à l'égard de la narration que je voudrais tenter de questionner ici.

\section{Rhétorique de l'exclusion}

\section{Le « clair obscur » de l'hérésie médiévale}

Si la spécificité du travail historique réside précisément dans l'attention portée aux documents, qui constituent les sources de son travail, la tâche du chercheur qui s'intéresse aux mouvements religieux hétérodoxes est pour le moins complexe. S'impose à lui, en effet, la nécessité de gérer un ensemble documentaire rare, hétérogène, et idéologiquement très orienté. Prenons le cas des phénomènes hérétiques du $\mathrm{XI}^{\mathrm{e}}$ siècle, souvent nommés " hérésies de l'An Mil » et analysés comme le signe des bouleversements intellectuels, voire psychologiques, occasionnés par l'arrivée du nouveau millénaire ${ }^{6}$. On note en effet une inflation de témoignages épars à partir du tout début du $\mathrm{XI}^{\mathrm{e}}$ siècle qui relèvent ponctuellement l'émergence de groupes hérétiques locaux ${ }^{7}$.

La difficulté que posent ces documents réside à la fois dans leur hétérogénéité (chronique, lettre, extrait de cartulaire), leur aspect ponctuel et leur grande partialité. L'œuvre de Raoul Glaber, par exemple, se montre en effet difficile à traiter : la dynamique millénariste qui guide son ouvre, et qui fait de lui la principale source sur les fameuses et controversées « peurs de l'An mil », tend à biaiser les événements qu'ils rapportent, à en accentuer la gravité : les mouvements hérétiques sont décrits comme les signes avant-coureurs de l'imminence du Jugement dernier. Sa rhétorique tient moins, d'ailleurs, à celle de l'historien, informant son lecteur de certains événements, que du conteur de légendes et de miracles. Son traitement de l'affaire de la communauté dissidente de Monforte le révèle parfai-

5 J'emprunte l'expression à Guy Lobrichon, «Le Clair-obscur de l'hérésie au début du XI ${ }^{\mathrm{e}}$ siècle en Aquitaine. Une lettre d'Auxerre ", dans Essays on the Peace of God: The Church and the People in Eleventh-Century France, éd. Thomas Head and Richard Landes, Special Issue of Historical Reflexions/Réflexions Historiques 14, 1987, p. 423-444.

6 Voir notamment, parmi de nombreuses publications sur un thème discuté, les travaux de Georges Duby, et ceux, plus récents, de Dominique Barthélémy, qui se répondent et se complètent : Georges Duby, L’An Mil, Paris, Gallimard, 1980 ; Dominique Barthélémy, L'an mil et la paix de Dieu. La France chrétienne et féodale (980-1060), Paris, Fayard, 1999.

7 Pour conserver un peu de limpidité au texte, je ne les détaille pas. Hilário Franco Júnior ("Les "abeilles hérétiques" et le puritanisme millénariste médiéval ", dans Le Moyen Âge, t. CXI, 2005, p. 71-93) en donne une liste qui tend à l'exhaustivité : «Ravenne (v. 970), Orléans (991 ?-1022), Vertus (997-1015), Sens (1009-1015), Mayence (1012), Aquitaine (1016-1028), Périgord (1017-1018), Toulouse (1018), Liège (1022), Arras (1025), Ravenne (1025), Charroux (1027), Monteforte (1028-1030), Châlons-sur-Marne (1043-1048)». On peut noter que toutes manifestent une aspiration évangélique, caractérisée par le refus de l'intercession de l’Église (rejet des sacrements) et un ascétisme rigoureux. 
tement ${ }^{8}:$ l'événementiel le cède à l'anecdotique, et l'historique au didactique ; l'enjeu de Glaber est d'édifier son lecteur, non de rapporter un épisode historique, et il conclut son récit en l'affirmant clairement : " Personne ne douta que cette vision ne lui eût été envoyée pour son instruction et pour la nôtre ».

La seconde source de renseignements sur la communauté de Monforte, Landolf Senior, offre au contraire un récit plus fourni et plus précis, plus riche en informations : son principal intérêt réside dans la transcription qu'il fait de l'interrogatoire supposé de Gérard, qui est à la tête de la communauté dissidente de Monforte, par l'évêque Aribert de Milan. Mais cette qualité, rare, ne doit pas nous abuser : le récit de Landolf se veut une apologie de l'évêché de Milan, et son objectif est de mettre en scène Aribert, de le parer des beaux habits du défenseur de l'orthodoxie. En offrant une description de l'évêque en action, il l'inscrit dans un récit qui laisse à la marge la communauté dissidente, la limitant au rôle passif du faire-valoir.

Il n'en est pas de même lorsqu'il s'agit pour l'auteur de signaler à la chrétienté l'imminence d'un danger hérétique, en décrivant la doctrine et les pratiques d'une communauté dissidente récemment repérée et que l'auteur a pu observer. Informer pour repérer et pour combattre : tel est l'objectif d'une grande quantité de documents d'origine ecclésiastique qui informent sur les mouvements dissidents au Moyen Âge; la forme la plus aboutie sur le plan didactique est sans doute les manuels d'inquisiteurs' ${ }^{9}$, qui donnent, sous l'aspect d'un catalogue, un certain nombre de traits caractéristiques de chacun des mouvements hérétiques. On pourrait estimer que ce projet implique la recherche d'une grande précision dans la définition des doctrines condamnées. Mais ce serait oublier que l'imaginaire clérical de l'hérésie est dominé par l'héritage antique, qui a légué aux autorités chrétiennes une taxinomie qui biaise l'appréhension des spiritualités dissidentes.

De ce point de vue, le clerc qui s'attache à décrire les hérésies le fait avec un outillage intellectuel dans lequel la figure d'Augustin occupe une place de choix : la lutte de l'évêque d'Hippone contre le manichéisme fait en effet figure de modèle au Moyen Âge. L'influence du De haeresibus, traité inachevé dans lequel Augustin s'attache à fournir un catalogue de toutes les hérésies connues dont il résume les traits principaux, a imposé une taxinomie plus ou moins arbitraire, où l'absence de rigueur dans la description doctrinale le mêle à la calomnie - les allusions aux mœurs scandaleuses des Manichéens sont nombreuses, et elles deviendront un topos de la littérature polémique -,

8 Il s'agit d'une communauté évangélique retranchée dans un castrum du Piémont italien. Raoul Glaber ne décrit pas les mœurs et les croyances de la communauté, mais raconte qu'une femme de la communauté avait introduit au chevet d'un chevalier le diable, qui lui propose la vie éternelle.

9 Un exemple de ce genre phare de la littérature hérésiologique à partir du XIII ${ }^{\mathrm{e}}$ siècle : Bernard Gui, Manuel de l'inquisiteur, éd. et trad. par G. Mollat, Paris, Les Belles Lettres, 1964. 
qui intervient dans la perception par les clercs du Moyen Âge des mouvements dissidents. Et lorsqu'Adémar de Chabannes parle de "Manichéens " en Aquitaine, il ne faut pas se laisser abuser par le terme : il n'est que le reflet de l'influence d'Augustin et de son combat contre l'hérésie majeure de son temps, le manichéisme, sur la culture ecclésiastique du Moyen Âge. En se servant de la taxinomie augustinienne, qu'ils répugnent à mettre au goût du jour, les clercs médiévaux peuvent ramener à du connu des croyances originales dont l'hétérodoxie leur était inquiétante et scandaleuse : ils pouvaient réduire ce scandale, en inscrivant leur lutte dans la descendance, rassurante, d'Augustin - rassurante parce que prestigieuse, rassurante aussi parce qu' elle laissait présumer une issue favorable, pareille à celle que connut Augustin.

On comprend ainsi aisément que la partialité dont font preuve les auteurs médiévaux qui nous donnent à lire les mouvements hérétiques pose problème à celui qui cherche à les appréhender dans leur singularité. D'abord parce que ces œuvres de combat manifestent une hostilité à l'endroit des croyances qu'ils décrivent : mais cette hostilité est tellement évidente qu'elles suscitent d'ellesmêmes une saine méfiance à leur égard. Plus grave est en revanche la déformation et l'uniformisation des croyances auxquelles aboutit nécessairement la perception biaisée des mouvements dissidents, de laquelle participe l'imaginaire clérical de l'hérésie : ce phénomène est plus perturbant non seulement parce qu'il se laisse moins aisément repérer, et nécessite une lecture fine de la littérature hérésiologique, dont il faut détacher les motifs qui, s'organisant en réseaux sémantiques, révèlent cet imaginaire de l'hérésie, mais aussi parce qu'il est plus difficilement contournable, car rares sont les documents qui permettent de contrôler les informations que ces œuvres de combat transmettent ; les documents issus des milieux dissidents font par exemple cruellement défaut ${ }^{10}$. On voit donc que l'hérésie médiévale apparaît en clair-obscur aux yeux de l'historien : omniprésente dans l'esprit des clercs, elle se dérobe à l'analyse fine et fouillée de son contenu.

10 On dispose à vrai dire à propos de l'hérésie " cathare ", d'un corpus hétérogène et fragmentaire de documents d'origine dissidente : l'Interrogatio Iohannis - apocryphe sans doute d'origine bogomile (du nom de ce courant dualiste apparu dans les Balkans au IX ${ }^{\mathrm{e}}$ siècle) - ; un traité philosophique rédigé au XII ${ }^{e}$ siècle par un dissident italien, Jean de Lugio, et intitulé Livre des deux principes; un " traité cathare " anonyme autant qu'hypothétique dans la mesure où il a été obtenu par des historiens contemporains en rapprochant les fragments d'un traité de réfutation anti-cathare rédigé par un polémiste catholique ; enfin deux exposés liturgiques. Si ces documents, par leur rareté même, sont d'un grand intérêt, ils ne peuvent être considérés comme un corpus présentant une vision cohérente du catharisme : leur hétérogénéité temporelle aussi bien que spatiale, et par conséquent culturelle, rend vaine cette tentative. Ils sont au contraire le reflet de la pluralité des formes que les mouvements dualistes ont pu prendre au cours du Moyen Âge, pluralité qu’a récemment mise en lumière Pilar Jimenez-Sanchez dans l'ouvrage issu de sa thèse (Les Catharismes. Modèles dissidents du christianisme médiéval, Rennes, Presses Universitaires de Rennes, 2008). Ces documents ont été réunis, et récemment réédités, dans une anthologie publiée par René Nelli et Anne Brenon, sous un titre éminemment contestable : Écritures cathares, Monaco, Éditions du Rocher, 2011. 


\section{Déconstruire le discours clérical : l'hérésie comme coproduction}

Ces difficultés ont généré depuis une quinzaine d'années environ une nouvelle lecture des sources hérésiologiques du Moyen Âge. Il faut mentionner à ce titre un recueil d'études réalisées dans le cadre du séminaire de Monique Zerner à Nice et réunies sous un titre provocateur : Inventer l'hérésie ?11 $\mathrm{Il}$ s'agit de centrer le regard sur les mécanismes de production du discours anti-hérétique par l'Église, et d'examiner l'hypothèse selon laquelle ce discours joue un rôle dans la détermination des croyances dissidentes. Le point d'interrogation qui suit l'expression "inventer l'hérésie " porte sur la validité du déplacement de signification autour du verbe "inventer » : si les clercs inventent l'hérésie, dans le sens où l'historien invente un document, c'est-à-dire qu'il en révèle l'existence à ses contemporains, peut-on dire qu'ils l'inventent comme on invente une histoire, dont on forge les personnages, les décors et l'intrigue? S'il n'est question pour personne de mettre en doute l'existence de communautés dissidentes au XII siècle, il semble néanmoins que l'hérésie médiévale et les formes marginales de spiritualité constituent le produit d'une coproduction entre ceux qui en sont les tenants et ceux qui, en les combattant, les donnent à voir et leur conferent une existence pour l'histoire.

Prenons un exemple, celui de l'hérésie la plus célèbre du Moyen Âge, la plus dangereuse pour les clercs, celle qui a le plus ébranlé l'histoire : le "catharisme $»^{12}$. Au cœur de la doctrine "cathare » se trouve le dualisme, qui pose l'existence de deux dieux créateurs, l'un bon l'autre mauvais. On a beaucoup glosé sur l'origine de ce dualisme, et jusque dans les années 1970, il ne faisait de doute pour personne qu'il s'agissait d'une réminiscence du manichéisme, transmis dans l'Europe occidentale via l'hérésie bogomile apparue dans les Balkans au $\mathrm{IX}^{\mathrm{e}}$ siècle. Cette tradition historiographique est aujourd'hui révisée ${ }^{13}$, et dans un article fondamental, Jean-Louis Biget a tâché d'envisager les causes endogènes du développement d'une théorie dualiste dans le monde latin, en choisissant

11 Monique Zerner (dir.), Inventer l'hérésie? Discours polémiques avant l'Inquisition, Nice, collection d'Études médiévales, 1998.

12 J'adopte ici par commodité ce terme problématique, problématique parce qu'il est largement un produit de l'orthodoxie, et plus encore de l'historiographie, sur laquelle la mémoire collective a greffé un ensemble de représentations qui n'ont rien d'historiques ; problématique aussi parce qu'il donne à voir un mouvement unique et centralisé, là où il faut au contraire percevoir la pluralité, la diversité et l'hétérogénéité, comme y invite d'ailleurs Pilar Jimenez-Sanchez. Pour une critique du terme, voir : Julien Théry, "L'hérésie des bons hommes. Comment nommer la dissidence non béguine et non vaudoise en Languedoc (XII - début du XIV ${ }^{e}$ siècle) ? ", dans Heresis, $\mathrm{n}^{\circ} 3637,2002$, p. 75-117.

13 On insiste en effet d'une part sur l'existence d'un dualisme latent dans la chrétienté latine, d'autre part sur la faiblesse des liens qui permettent d'établir une filiation historique avec le manichéisme antique. Voir notamment : Pilar Jimenez-Sanchez, op. cit. ; Uwe Brunn, Des contestataires aux Cathares. Discours de réforme et propagande antihérétique dans les pays du Rhin et de la Meuse avant l'Inquisition, Paris, Institut d'Études Augustiniennes, 2006. 
comme cadre d'étude le Languedoc de la fin du XII ${ }^{\mathrm{e}}$ siècle $^{14}$. Biget propose de renverser la réflexion, et de voir dans le dualisme non pas la cause de l'anticléricalisme des dissidents, mais au contraire son effet, sa conséquence immédiate. Il montre en effet, en retraçant la chronologie des accusations contenues dans les traités polémiques languedociens, que jusqu'aux environs de l'année 1165, il existe un courant anticlérical et évangélique - et anticlérical, parce qu'évangélique - ; le dualisme n'intervient que dans un second temps, et aboutit à la formation d'un courant spécifique - identifié par les historiens sous le nom de " catharisme ». Cette reconstruction chronologique permet de faire l'économie de la théorie de l'importation exogène du dualisme, en montrant que le terrain lui était préparé : en cela, le dualisme est non pas la cause de l'anticléricalisme des dissidents, mais au contraire sa conséquence. Tout invite à penser en effet que le dualisme est une manière de radicaliser le rejet de l'Église en l'assimilant à l'Église du diable, qui, dans la cosmogonie dualiste, est le maître de ce monde terrestre. Ainsi, le dualisme se comprend aisément, sans recourir à l'hypothèse d'une influence extérieure, en considérant que le dualisme permet de « disqualifier ontologiquement » (Biget) les clercs.

Une fois que les traces d'un dualisme sont repérées dans le discours des dissidents languedociens, les polémistes orthodoxes mettent en place une rhétorique de la dénonciation et de l'identification des hérétiques à des formes antiques d'hérésies, induisant ainsi une parfaite continuité entre Manichéens et dissidents languedociens. Et l'identification des dissidents à l'image que leurs adversaires leur offrent d'eux-mêmes est une manière de radicaliser davantage leur refus de l'Église. La sociologie propose maints exemples de ce processus complexe et paradoxal de constitution des formes minoritaires et marginales de culture, qui se construisent une identité collective en épousant, et en assumant, au point de le revendiquer, le regard, pourtant hostile, réducteur et globalisant, de l'autre. Ce jeu de regards complexe participe par exemple de la constitution des cultures «black» ou " gay" : ainsi en va-t-il de la constitution de ce que Jean-Loup Amselle nomme les « logiques métisses ${ }^{15}$.

C'est à ce titre qu'il faut parler de coproduction : la littérature de combat mise en œuvre avec force par les clercs à partir du XII ${ }^{\mathrm{e}}$ siècle a participé à créer la réalité qu'ils décrivaient et dénonçaient. La narration a ainsi une dimension performative, comme tout acte de langage, mais avec des mécanismes spécifiques.

14 Jean-Louis Biget, "L'anticléricalisme des hérétiques d'après les textes polémiques ", dans Cahiers de Fanjeaux, no 38, 2003, p. 405-445.

15 Jean-Loup Amselle, Logiques métisses. Anthropologie de l'identité en Afrique et ailleurs, Paris, Payot, 1990. 


\section{À quoi sert l'hérésie?}

Cette participation de l'orthodoxie à la mise en récit des communautés dissidentes du Moyen Âge n'est pas sans raison, elle n'est pas que la conséquence incidente d'une œuvre de combat. Et il faut se demander maintenant pour quelles raisons l'Église a inventé l'hérésie, l'a inscrite dans un récit et a répandu son image. On peut, à ce titre, avancer deux séries d'hypothèses.

La première recouvre la question de la marginalité sociale et religieuse ; il faut en effet considérer qu'elle joue un rôle social éminent dans la cohésion d'une communauté, dans la mesure où les marges d'un ensemble social permettent, en creux, de définir les traits constitutifs du groupe : l'exclusion est en cela un principe d'unité. La société chrétienne du Moyen Âge assure sa cohésion autour de deux ennemis : d'abord, l'ennemi de l'extérieur, l'Infidèle, qui suit la loi de Mahomet et refuse le Christ et contre lequel la guerre est juste et sanctifiante ; ensuite l'ennemi de l'intérieur, et à ce titre d'autant plus dangereux qu'il menace de corrompre la société chrétienne en s'immisçant dans ses rouages, en se parant de ses caractéristiques. L'imaginaire chrétien de l'hérétique est d'ailleurs marqué par l'accusation récurrente du travestissement, de la dissimulation de l'hétérodoxie sous des habits orthodoxes : le sursaut évangélique auquel appellent les communautés dissidentes depuis le XIe siècle jusqu'à l'épisode cathare - et, sous une autre forme, aux $\mathrm{XIV}^{\mathrm{e}}-\mathrm{XV}^{\mathrm{e}}$ siècles - est ainsi décrit comme un habile procédé de propagande, destiné à cacher les mœurs forcément scandaleuses qui caractérisent ces êtres nécessairement scandaleux. Ce faisant, l'Église retourne contre les dissidents ce que ces derniers évoquent précisément à son propos : avarice, conduite sexuelle scandaleuse, hypocrisie, etc. Dans cette attaque contre ceux qui, rompant l'unité de la foi, sèment la discorde et font advenir le scandale ${ }^{16}$, l'Église trouve un ferment d'unité, un facteur de cohésion, qui lui permet de préciser les contours de la société chrétienne. D’autant que le christianisme a connu, à partir du XIII e siècle, des évolutions fondamentales dont on peut penser qu'elles sont le fruit de la contestation dissidente ${ }^{17}$, preuve des liens étroits qui unissent l'orthodoxie et l'hérésie, qui sont davantage à penser sur le mode dialectique qu'en termes d'opposition irréductible.

16 Or, selon Matthieu 18, 7 : «Malheur à celui par qui le scandale arrive ! ».

17 On peut résumer ces innovations en reprenant les expressions d'André Vauchez qui parle de " christianisme d'incarnation" ("Le christianisme roman et gothique ", dans Jacques Le Goff et René Rémond (dir.), Histoire religieuse de la France. Tome II, Paris, Seuil, 1988, p. 394) pour caractériser la valorisation de l'humanité du Christ, et de " tournant pastoral " ( Le tournant pastoral de l'Église en Occident ", dans Jean-Marie Mayeur, Charles Pietri, André Vauchez et Marc Venard (dir.), Histoire du christianisme. Tome V. Apogée de la papauté et expansion de la chrétienté (1054-1272), Paris, Desclée, p. 737-774), pour insister sur la place nouvelle prise par la prédication en réponse aux succès de la pastorale dissidente, permise notamment par l'essor des Ordres mendiants. 
On comprend mieux ainsi l'utilité des formes marginales de croyance pour une orthodoxie qui cherche d'une part à assurer à la cohésion de la communauté des croyants et d'autre part à adapter son discours aux attentes des fidèles : en cela, la marginalité est au cœur de la constitution des centres, des lieux de pouvoir qui pourtant les condamnent à la marginalité, et c'est pourquoi leur étude et leur prise en compte est si capitale pour l'historien ${ }^{18}$.

Mais l'hérésie s'inscrit aussi, et peut-être surtout, dans l'économie des pouvoirs de la société médiévale, et on peut constater ce rôle politique de l'hérésie à deux niveaux de temporalité. À court terme, la question hérétique s'ancre dans les relations géopolitiques de l'Occident médiéval, et JeanLouis Biget a très bien montré ${ }^{19}$ comment la focalisation de la polémique anti-hérétique sur le Languedoc tient d'abord à la situation délicate du comté de Toulouse au XII ${ }^{e}$ siècle, pris en tenaille entre des puissances rivales (il constitue une zone tampon entre le duché d'Aquitaine à la tête duquel se trouve le roi d'Angleterre, et le royaume de France). L'accusation d'hérésie lancée sur un territoire constitue un excellent prétexte d'intervention, dont l'auteur peut tirer un bénéfice territorial et politique tout en s'inscrivant dans la lutte contre l'hérésie au service de l'Église, tâche légitime et prestigieuse. Les cathares languedociens ont donc été victimes d'intérêts géopolitiques qui, pour une large part, ne regardaient pas la religion : en cela, la première cause de la Croisade amorcée en 1209 est à chercher dans la dynamique expansionniste de la couronne capétienne, soucieuse de mettre au pas les velléités de la maison de Toulouse et des seigneurs méridionaux.

Mais si l'on déplace le curseur chronologique, on s'aperçoit que la répression anti-hérétique, qui s'appuie d'abord sur la mise en récit des communautés dissidentes et ensuite sur une répression armée et judiciaire, s'inscrit dans un vaste mouvement de l'histoire des pouvoirs dans l'Europe médiévale, et dans lequel l'historien anglais Robert Ian Moore a perçu la mise en place d'une "société de persécution ${ }^{20}$. Il montre en effet comment la genèse de l'État moderne s'est appuyée sur une politique d'exclusion, des hérétiques, des Juifs et des lépreux notamment, dans une dynamique de renforcement et de

18 Jean-Claude Schmitt le relevait déjà à la fin des années 1970 dans l'ouvrage manifeste de la Nouvelle histoire ("L'histoire des marginaux ", dans Jacques Le Goff, Roger Chartier et Jacques Revel (dir.), La Nouvelle Histoire, Paris, La Bibliothèque du C.E.P.L., 1978, p. 344-369) : "À partir du centre, il est impossible d'embrasser du regard une société toute entière et d'écrire autrement son histoire qu'en reproduisant les discours unanimistes des détenteurs du pouvoir. La compréhension jaillit de la différence : il faut pour cela que se croisent des angles de vue multiples, qui révèlent de l'objet - considéré cette fois à partir de ses marges ou de l'extérieur autant de faces différentes l'une à l'autre cachées. "

19 Jean-Louis Biget, «Hérésie, politique et société en Languedoc (1120-1320) », dans Jacques Berlioz (dir.), Le Pays cathare, Paris, Seuil, 2000, p. 17-80.

20 Robert I. Moore, La Persécution. Sa formation en Europe (X'e-XIII siècles), Paris, 10/18, 1997 [1991]. 
centralisation des pouvoirs. Pour le XIII ${ }^{e}$ siècle, il est évident que la répression des communautés hérétiques sert l'affirmation d'une " théocratie pontificale $»^{21}$ en construction, et dont la mise en place de l'Inquisition - juridiction extraordinaire ne relevant que du pape - est un ressort fondamental.

Ainsi, l'Église médiévale a décrit les mouvements dissidents en les inscrivant dans un récit empreint d'un imaginaire spécifique, hérité pour une large part de l'Antiquité, et qui biaise fondamentalement la description des croyances hétérodoxes. Les récits parvenus à nous sont le fruit d'une coproduction entre l'orthodoxie, qui condamne et qui exclut, et l'hérésie, qui voit dans l'image que lui renvoient ses adversaires une manière d'exister, les deux ensembles entretenant ainsi une relation dialectique. Le premier travail de l'historien est donc de déconstruire ces récits et ces discours, en s'attachant à étudier les rouages et les mécanismes de leur production. Mais ce travail ne peut suffire, et il n'est pas question de renoncer à écrire, à décrire, les formes marginales de spiritualité et leur ancrage dans le monde social, à charge pour le chercheur de ne pas reproduire les discours qu'il a déconstruits, et surtout de se méfier des récits qu'il forge, qui s'imposeraient avec l'argument de la nouveauté et le masque de la scientificité, rendant impossible toute appréhension véritable des communautés dissidentes.

\section{Dire l'autre : quel discours pour l'historien?}

Les lignes qui vont suivre proposent d'une part d'envisager, à partir de l'exemple des hérésies médiévales, les écueils liés au manque de distance ente l'historien et son objet, qui aboutit à augmenter la tentation narrative de l'écriture historique et à laisser à la marge le cour de son objet, et suggérer d'autre part une voie pour dire les croyances marginales, et décrire les croyances des autres.

\section{Poétique : variations sur l'héroïsme occitan}

Sans entrer dans de savantes définitions épistémologiques des concepts d'histoire et de mémoire, on peut dire que la mémoire constitue l'appropriation collective et contemporaine d'un événement passé : il s'agit, en quelque sorte, d'écrire l'histoire au présent, c'est-à-dire de ne pas mettre le passé au

21 Cette question est au cœur de travaux récents, comme ceux de Julien Théry : Julien Théry, «Le gouvernement romain de la Chrétienté autour de 1206 : Innocent III et les débuts de la théocratie pontificale ", dans Dominique avant les dominicains, Paris, Le Cerf, coll. " Mémoire dominicaine ", 2007, p. 33-37. Voir aussi une synthèse récente sur le XIII" siècle : Julien Théry, "Le triomphe de la théocratie pontificale du III concile du Latran au pontificat de Boniface VIII (1179-1303) ", dans Marie-Madeleine de Cevins et Jean-Michel Matz (dir.), Structures et dynamiques religieuses dans les sociétés de l'Occident latin (1179-1449), Rennes, Presses Universitaires de Rennes, 2010, p. 17-31. 
cœur du travail, mais d'en faire un détour, un support pour un discours qui porte avant tout sur le présent. Ce faisant, la mémoire inscrit le passé dans un récit où il n'a pas sa place, et où il n'est admis qu'à titre temporaire.

De ce point de vue, l'histoire des hérésies méridionales a été, et continue d'être l'objet d'une mémoire vive et richement entretenue. Ce phénomène est bien connu aujourd'hui ${ }^{22}$ : il faut en dire deux mots ici, sans retracer l'histoire de cette mémoire, car il a influencé, et influence encore, la perception des hérésies médiévales.

L'exemple le plus caractéristique de cette influence du présent sur l'écriture du passé est sans doute à situer dans les années 1960-1970 : on voit alors s'épanouir dans le Midi toulousain le mouvement occitaniste, qui revendiquait l'existence d'une identité culturelle spécifique menacée. Cet état d'esprit a suscité un certain nombre de travaux, qui ont donné à voir le XIII ${ }^{\mathrm{e}}$ siècle et l'histoire du catharisme toulousain comme le moment clef du basculement culturel du Midi. Dans cette lecture, le catharisme est perçu comme une spécificité méridionale ${ }^{23}$, qui serait le reflet d'une culture authentique ${ }^{24}$. Cette hypothèse induit celle d'une large adhésion de la société méridionale à la dissidence, et donne à voir l'image d'un peuple uni dans la résistance à l'envahisseur : l'Église, qui veut imposer la religion romaine, et la royauté capétienne, qui cherche à introduire la culture française ${ }^{25}$ dans le Midi. Il faut noter la proximité des mouvements de décolonisation, dont sont proches les représentants de cette " aile gauche " du mouvement occitan ; le schéma de la résistance à l'occupation allemande n'est pas non plus très loin, et irrigue l'imaginaire de la révolte et de l'insoumission dont est empreinte la lecture des événements militaires. L'œuvre monumentale de Michel Roquebert est ainsi marquée par cette lecture des événements politiques : ce que l'historien entend raconter ici, c'est "l'épopée cathare ${ }^{26}$, retracée chronologiquement ; et

22 La contribution la plus récente, et peut-être la plus riche, à la question de la mémoire occitane du catharisme est l'ouvrage de René Soula, issu de sa thèse d'anthropologie historique : René Soula, Les Cathares, entre légende et histoire, Toulouse, Institut d'Études Occitanes, 2005.

23 Ce qui est paradoxal, notons le rapidement, c'est que cette insistance sur les liens entre le mouvement dissident et la culture occitane n'exclut pas l'inscription du catharisme dans la filiation du manichéisme, ni même son implantation dans des zones culturellement aussi différentes du Midi que l'était par exemple le pays rhénan.

24 René Nelli, un des plus fameux représentant de ce courant écrit ainsi (Les Cathares, Paris, Marabout, 1972, p. 9) : «Dans le midi de la France, la doctrine et le mode de vie cathares ont traduit l'âme, la sensibilité profonde de tout un peuple. Ce fut le produit spontané, naturel, d'une certaine manière de voir et de ressentir le monde, propre à cette société occitane si différente de ce qu'on pouvait alors appeler la société française. [...] Pour qu'une religion originale se soit ainsi formée, il fallait qu'il y eût un terrain social favorable, une civilisation originale ».

25 Jusqu'au XIVe siècle au moins, on nommait « Français », les seuls ressortissants de l'actuelle Île de France. Sur la question de l'émergence de l'identité française, qui intervint véritablement dans le contexte de la guerre de Cent ans, voir : Colette Beaune, Naissance de la Nation France, Paris, Gallimard, 1985.

26 Michel Roquebert, L'épopée cathare, 5 vol., Toulouse, Privat, 1970-1998. 
le récit qu'il entreprend est bien celui de la résistance occitane à "l'invasion " - c'est le titre du tome I - française. La bataille de Muret, qui se solda par la victoire des Croisés emmenés par Simon de Montfort devant le roi d'Aragon et le comte de Toulouse, est ainsi perçue comme une « dépossession »-c'est le titre du tome II -, dépossession des méridionaux de leur identité commune qui pouvait trouver une institutionnalisation dans le rapprochement politique du Midi et de la couronne d'Aragon. L'enjeu est alors d'offrir un récit qui se veut fidèle et historique des événements militaires, propre à donner aux Occitans contemporains une conscience d'eux-mêmes dans l'histoire, une profondeur historique qui légitime la revendication de leur identité culturelle singulière : le récit de Roquebert est en cela un récit fondateur ${ }^{27}$. S'il est un moyen pour transmettre une connaissance exhaustive des événements, la mise en récit vient en la circonstance imposer un discours idéologique qui participe à occulter l'authenticité d'une spiritualité singulière. En faisant des seigneurs méridionaux des " primitifs de la révolte ", pour reprendre l'expression d'Eric Hobsbawm, l'historien tend à amincir la frontière entre le narratif fictionnel et le narratif historique, et la distinction de Rancière ${ }^{28}$, qui distingue nettement les deux catégories de récit, tend à devenir caduque. Il ne s'agit pas ici d'opposer de manière tranchée et inconciliable le discours scientifique, qui serait du côté du réel, et le discours narratif, du côté de la fiction ; il va de soi que le récit, et même la fiction, ont pour l'historien une portée heuristique ${ }^{29}$, mais cela à condition qu'il ne s'agisse que d'un détour : lorsque le cœur du travail n'est plus l'analytique ou l'interprétatif, mais le narratif, on perd de vue l'objet de l'historien, qui est de donner à voir la singularité du passé - et elle seule.

27 Ces remarques n'empêchent évidemment pas que la reconstitution des événements militaires qui est le cœur de son travail répondent aux exigences d'un travail historique

28 Jacques Rancière distingue en effet l'écriture historique de l'écriture romanesque par " l'ensemble des procédures littéraires par lesquelles un discours se soustrait à la littérature, se donne un statut de science et le signifie" (Les Mots de l'histoire. Essai de poétique du savoir, Paris, Seuil, 1992, p. 21).

29 Il faut méditer sur ce point les nombreuses réflexions historiographiques de Carlo Ginzburg, qui me semble proposer les pistes les plus intéressantes pour dépasser la stérilité du débat entre positivistes voués au culte de la vérité, et relativistes radicaux : "Contre la tendance du scepticisme postmoderne à estomper la frontière qui passe entre les récits de fiction et les récits historiques au nom de l'élément constructiviste qui permet de les réunir, je proposais alors de considérer leur rapport comme une lutte pour la production de la réalité », une lutte qui serait " un conflit fait de défis, d'emprunts réciproques, d'hybrides ", et " pas une guerre de tranchées " (Carlo Ginzburg, Le Fil et les traces. Vrai faux fictif, Lagrasse, Verdier, 2010, p. 9). Il faudrait prendre davantage de temps pour le démontrer, mais on peut signaler qu'un des mérites de l'apport de Ginzburg réside dans l'abandon de la recherche d'une prééminence hiérarchique entre la fiction et l'histoire, dont les mérites réciproques seraient évalués et distingués à l'aune de leur capacité à dire le monde, sa vérité. Il lui substitue une vision horizontale et concurrentielle : en ce sens, la fiction est utile à l'historien en tant que " mise à l'épreuve du réel ». Sur les réflexions de Ginzburg, voir celles de Rancière : "De la vérité des récits au partage des âmes ", dans Critique, n 769-770, 2011, p. $474-484$. 
Or, s'agissant des travaux auxquels je faisais allusion, leurs conclusions sont aujourd'hui remises en cause. L'hypothèse d'une " civilisation originale " (René Nelli) épousant spontanément le catharisme s'effondre lorsque l'on s'aperçoit que la dissidence a manifestement été minoritaire en Languedoc ${ }^{30}$. De même, l'image d'un peuple résistant unanimement à l'envahisseur ne résiste guère à l'analyse, qui prouve que des dissensions ont existé au sein de la population méridionale, et que l'attitude des bourgeois toulousains, par exemple, ne répond ni à un sentiment patriotique, ni à une conviction religieuse, mais pour une large part à un opportunisme politique et économique ${ }^{31}$. Enfin, les qualités de tolérance prêtées aux dissidents, victimes de l'intolérance ecclésiastique, porteurs d'une spiritualité pure et originelle, n'est appuyée sur aucun fait historique - si ce n'est leur répression même - et se situe, en cela, en dehors du discours de l'historien ${ }^{32}$.

Ainsi, s'agissant du catharisme, qui a été investi par des facteurs politiques et affectifs particulièrement vifs - qui expliquent d'ailleurs la violence avec laquelle les travaux récents sont accueillis ${ }^{33}-$, il est indéniable que la dimension narrative du discours de l'historien a contribué à importer un bagage idéologique tendant à obscurcir la réalité historique.

\section{L'individuel et le collectif: affronter l'universelle singularité de la croyance}

Néanmoins, il serait par trop réducteur d'opposer terme à terme la catégorie du récit et la catégorie du réel, en confiant à celui-ci une signification rationnelle et, partant, étroite. Le réel est au contraire à percevoir dans sa complexité, et il invite à multiplier les tentatives d'approche et les stratégies d'écriture et d'interprétation. C'est ce qu'il faut tenter de faire maintenant : brosser les contours de ce qui est à la disposition de l'historien qui cherche à manier avec maîtrise la dimension narrative, nécessairement narrative ${ }^{34}$, de son écriture, pour affronter la pluralité du réel.

30 Les tentatives d'évaluations quantitatives de Jean-Louis Biget ou de Jean-Loup Abbé le montrent : Jean-Louis Biget, Hérésie et Inquisition dans le Midi de la France, Paris, Picard, 2007, p. 47 ; Jean-Loup Abbé, "La société urbaine languedocienne et le catharisme au XIII siècle : le cas de Limoux ", dans Patrick Boucheron et Jacques Chiffoleau (éd.), Religion et société urbaine au Moyen Âge. Études offertes à Jean-Louis Biget par ses anciens élèves, Paris, Publications de la Sorbonne, 2000, p. 119-139.

31 Jean-Louis Biget, "Hérésie, politique et société en Languedoc (1120-1320) ", dans Jacques Berlioz (dir.), Le Pays cathare, Paris, Seuil, 2000, p. 17-80.

32 Jean-Louis Biget cite à ce sujet un aphorisme de Cioran (" Ayons pour toute victime une pitié sans illusion ") et souligne que rien n'indique que, si le rapport de force avait tourné en la faveur des dissidents, ceux-ci n’aient pas fait preuve de davantage de tolérance. Ce concept moderne est d'ailleurs anachronique pour la période qui nous occupe.

33 Voir notamment la vigueur des propos, qui dépassent parfois les formes du débat scientifique, tenus dans cet ouvrage collectif: Martin Aurell (dir.), Les Cathares devant l'histoire. Mélanges pour Jean Duvernoy, Cahors, L'Hydre, 2005.

34 C'est en effet sur l'idée de l'omniprésence du récit dans l'écriture que Barthes ouvre un article fameux : «Introduction à l'analyse structurale des récits », dans Communications, 8, 1966, p. 1-27. 
S'agissant des dissidences médiévales, les exemples abondent du profond renouvellement encore en cours, et qui s'inscrit dans la continuité du travail de déconstruction du discours clérical sur l'hérésie que nous évoquions. On peut ainsi mentionner l'effort sémantique et conceptuel, qui a amené certains historiens à remettre en cause les mots du lexique de l'hérésie. Il s'est d'abord agi de déconstruire les appellations médiévales ${ }^{35}$, puis les catégories des historiens, en critiquant le terme "catharisme ${ }^{36}$, et en reconsidérant l'usage du mot "hérésie ", trop lié au discours de l'Église, auquel s'est peu à peu substitué le terme plus neutre de "dissidence $»^{37}$. Ces tentatives ne sont pas le reflet d'un nominalisme excessif : les mots ont un sens, et ils peuvent, au-delà de ce qu'ils dénotent, véhiculer un imaginaire, et c'est en cela qu'ils engagent une représentation du monde.

Ces réflexions invitent à évoquer une voie de recherche qui reste à explorer. Le principal mérite de ces redéfinitions réside dans ce qu'elles permettent de complexifier la vision globale et unifiante, et en cela naïve, qu'induit le terme d'hérésie. De ce point de vue, il faut se méfier des " unités fictives " dénoncées par Michel Foucault dans des pages fameuses de L'Archéologie du savoir ${ }^{38}$. L'enjeu de la recherche actuelle est de se défaire des focales léguées par les clercs du Moyen Âge et de saisir dans leur pluralité les croyances hétérodoxes. Si sur le plan politique, la remise en cause de la vision unitaire de la population méridionale dans son soutien aux dissidents et dans son adhésion à leur discours est aujourd'hui admise, il n'en va pas de même lorsqu'il s'agit d'affronter l'individualité de la croyance. On a longtemps reproché aux ethnologues de généraliser les croyances des populations qu'ils s'attachaient à décrire, et l'anthropologie s'est aujourd'hui dotée d'un outillage conceptuel et méthodologique permettant de surmonter cette difficulté. L'introduction de ces procédures à l'histoire des dissidences médiévales est en revanche un chantier fécond à entreprendre.

La difficulté pour l'historien réside dans l'affranchissement nécessaire qu'il lui faut effectuer à l'égard des catégories héritées, pour une part, du discours clérical et pour une autre, la plus redoutable, de la tradition de l'histoire des religions, qui s'est longtemps donné pour tâche de repérer des courants

35 Voir notamment: Monique Zerner, «Du court moment où on appela les hérétiques des "bougres". Et quelques déductions ", dans Cahiers de civilisation médiévale, no 128, 1989, p. 305-324; et Jean-Louis Biget, "Les "Albigeois" : remarques sur une dénomination", dans Monique Zerner (dir.), Inventer l'hérésie ? Discours polémiques et pouvoirs avant l'Inquisition, Nice, Centre d'Études Médiévales, 1998, p. 219-255.

36 Voir : Julien Théry, art. cit. ; et Mark Gregory Pegg, "Historiographical essay. On Cathars, Albigenses and Good Men of Languedoc ", dans Journal of Medieval History, n 27, 2001, p. 181-195.

37 Voir notamment : Jean-Louis Biget, Hérésie et Inquisition dans le Midi de la France, Paris, Picard, 2007.

38 Michel Foucault, L'Archéologie du savoir, Paris, Gallimard, 1969, p. 33-46. 
de pensée théologique cohérents, formant un système de pensée. Or cette démarche réduit considérablement le champ de l'analyse, puisqu'il ne peut s'agir d'investiguer que les formes savantes, écrites, de la pensée religieuse. Ce faisant, elle laisse à penser que seuls les théologiens socialement reconnus comme tels et formés en ce sens, sont dignes d'exister dans l'histoire des croyances et sont seuls capables d'innovation religieuse.

Cette théorie anthropologique implicite présente le double inconvénient d'être discutable sur le plan philosophique et de n'être jamais démontrée, éprouvée ni débattue sur le plan scientifique. Or, les indices existent qui révèlent la grande pluralité des croyances, leur circulation et leurs confrontations, laissant induire une grande latitude dans la réception et l'assimilation du message religieux. Percevoir ces indices nécessite que l'historien leur ouvre son regard, qu'il se laisse étonner, surprendre et qu'il s'intéresse à ce que Carlo Ginzburg nomme des " grains de sable ». Il résume en effet dans un livre d'entretiens ce qui pourrait constituer un véritable manifeste : «il faut partir du grain de sable dans l'engrenage, [car] si on prend les règles pour point de départ, on risque de tomber dans l'illusion qu'elles fonctionnent " ${ }^{39}$. L'intérêt de cette phrase réside moins dans l'appel pour une micro-histoire - qui ne doit pas nous étonner chez Ginzburg que dans le fait que cette démarche historique s'appuie implicitement, mais solidement, sur une théorie globale des rouages sociaux, qui insiste sur l'idée que le fonctionnement des normes est une «illusion ». S'ouvrir aux grains de sable nécessite donc de s'affranchir des discours produits par les instances de pouvoir, pour reprendre une phraséologie foucaldienne, pour saisir ce qui peut leur résister et les remettre en cause.

Il semble pertinent de fonder sur ces bases une théorie de la croyance, autrement dit des mécanismes de l'adhésion ou du rejet à des énoncés religieux, qui permettent de penser l'histoire des formes marginales de spiritualité au Moyen Âge. Jean-Pierre Albert a ainsi ouvert une voie, en proposant, dans le cadre de son " anthropologie au conditionnel $»^{40}$ et sur le mode expérimental, d'éclairer ces phénomènes en recueillant les fruits de l'anthropologie $\operatorname{cognitive}^{41}:$ de ces travaux, il garde l'idée que les rouages de la croyance ont des assises biologiques qui, sans déterminer aucunement leur contenu, offrent des procédés cognitifs qui sont partout et toujours disponibles. L'enjeu est

39 Carlo Ginzburg, Un seul témoin. Texte présenté par Fabien Jobard et suivi d'un entretien avec Philippe Mangeot, Paris, Bayard. 2007, p. 78-79.

40 Jean-Pierre Albert, "Histoire et anthropologie : convergences et spécificités ", dans L'Atelier du Centre de recherches historiques, 2010, [en ligne] : http://acrh.revues.org:index1926.html, consulté le 8 février 2011.

41 Je résume sous cette étiquette commode les travaux Dan Sperber ( $L a$ Contagion des idées. Théorie naturaliste de la culture, Paris, Odile Jacob, 1996) ou de Pascal Boyer (Et l'homme créa les dieux. Comment expliquer la religion, Paris, Gallimard, coll. " Folio », 2003 [2001]), qui cherchent des fondements naturels aux phénomènes religieux. 
ainsi de mettre en lumière des modèles intervenant dans la constitution des croyances, permettant d'expliquer et de comprendre de manière rationnelle ces " croyances apparemment irrationnelles ${ }^{42}$. C'est nourri de ces acquis que JeanPierre Albert a proposé une lecture nouvelle et novatrice du registre d'Inquisition de Jacques Fournier ${ }^{43}$ : ce document, qui donne à lire une centaine de dépositions d'habitants de la haute Ariège au début du XIVe siècle, se distingue d'une part par la grande place qu'il accorde à la parole des accusés, et d'autre part par son ouverture à des formes marginales de dissidence, qui donnent à voir des types d'hétérodoxies qui ne rentrent pas dans les cases des manuels d'Inquisition - ni dans ceux, pourtant mieux informés, de l'histoire des religions. L'analyse ${ }^{44}$ de Jean-Pierre Albert insiste sur la primauté du «bricolage » dans la constitution des croyances : les individus font dialoguer les énoncés religieux qui leur ont été transmis, les confrontent avec les attentes du sens commun, pour se former leurs représentations religieuses, qui constituent le résultat de ce " tricotage intime ", pour reprendre la belle formule de Julien Gracq, qui est au cour de la construction des croyances. On comprend alors que ce mécanisme, mis en lumière par les sociologues des religions ${ }^{45}$ n'est pas une spécificité d'un monde sécularisé et désenchanté, mais constitue un procédé cognitif partout et toujours disponible. Le cas du meunier frioulan Menocchio, étudié par Carlo Ginzburg ${ }^{46}$, est à ce titre exemplaire : à travers son procès pour hérésie, il montre que ce meunier que rien ne prédisposait à la théologie, a construit un ensemble de représentations religieuses irréductibles aux courants dominants de la théologie du XVI siècle, et qui constituent en cela de véritables innovations religieuses.

La tentative de reconstruction d'un parcours spirituel, la mise à plat des fils qui ont participé à la mise au point d'énoncés religieux alternatifs, permet ainsi d'éviter le piège du récit narratif et descriptif, qui emprunte ses codes à la fiction romanesque. Cette démarche se fonde sur le constat de la grande diversité du réel, qui résiste aux catégories globalisantes, et propose ainsi de disposer le regard de l'historien au niveau microscopique de l'individuel. Si les mécanismes de la croyance répondent à des schémas sans doute universels, leur approche nécessite ce détour par l'observation et la compréhension des logiques locales. Ce faisant, cette manière de contourner la dimension potentiellement fictionnelle de la mise en récit atomise les communautés sociales

42 Dan Sperber, Le Savoir des anthropologues, Paris, Hermann, 1982, p. 49 et sqq.

43 Le registre d'Inquisition de Jacques Fournier, évêque de Pamiers (1318-1325), édité par Jean Duveroy, Toulouse, Privat, 1965. C'est le document sur lequel s'appuie le Montaillou de Leroy-Ladurie.

44 Voir notamment : Jean-Pierre Albert, "Croire et ne pas croire. Les chemins de l'hétérodoxie dans le registre de Jacques Fournier ", dans Heresis, n³9, 2003, p. 91-107 ; et id., " Hérétiques, déviants, bricoleurs. Peut-on être un bon croyant ?", dans L'Homme, n 173, 2005, p. 75-95.

45 Par exemple : Danièle Hervieu-Léger, La Religion en mouvement : le pèlerin et le converti, Paris, Flammarion, 1999.

46 Carlo Ginzburg, Il formaggio et i vermi. Il cosmo di un mugnaio del 500, Turin, Einaudi, 1976. 
et religieuses, en extrayant des individus qui servent de focale au regard de l'historien. Si les grands récits sur les hérésies médiévales mettaient en avant le collectif, au point d'en faire l'acteur principal, cette anthropologie des croyances nécessite, semble-t-il, de mettre à mal cette globalité, renvoyant son interprétation dans un second temps de l'analyse.

À l'heure du bilan, un constat s'impose : lorsqu'on étudie l'historiographie des dissidences méridionales, le couple narration/lien social est à percevoir sous l'angle de l'opposition hostile. Les développements récents de l'historiographie des hérésies médiévales ont indéniablement permis de mieux comprendre le fonctionnement discursif de la mise en récit des formes marginales de spiritualité : le fonctionnement discursif, c'est-à-dire la façon dont les récits qui intègrent les dissidences religieuses au Moyen Âge véhiculent un imaginaire tendant à reléguer, derrière la narration, l'authenticité d'une croyance. La déformation est d'autant plus frappante lorsqu'il s'agit de penser une communauté religieuse dans son ensemble : la complexité du lien social se perd dans les méandres d'une enveloppe narrative étroite, qui importe tout un bagage de représentations et de codes. Une solution réside dans la micro-analyse, dans l'attention portée à ces grains de sable dont l'assemblage forme les dunes enfouies que l'on cherche péniblement à saisir et à appréhender : de ce point de vue, l'anthropologie offre des cadres conceptuels et méthodologiques qui permettent d'élucider des logiques culturelles locales et spécifiques. La question n'est pas tellement celle de la place des liens qui unissent les membres d'une communauté sociale et religieuse que celle des mécanismes d'interaction entre différents individus, entre différents sujets individuels, dont il faut chercher à percevoir le fonctionnement cognitif à l'œuvre dans la constitution des représentations religieuses : en cela, cette démarche scientifique se fonde sur un humanisme individualiste, qui accorde à chacun la pleine qualité d'individu.

Matthieu Montantou M2 Histoire médiévale Université Michel de Montaigne Bordeaux 3 matthieu.montantou@hotmail.fr 


\section{Résumé}

L'enjeu de ce travail est de proposer quelques pistes de réflexions pour cerner le récent renouvellement de l'historiographie des dissidences médiévales. Il s'agit en effet de s'interroger sur les ressources à la disposition des historiens pour saisir les deux niveaux de réalité historique : car il semble nécessaire de distinguer clairement les phénomènes eux-mêmes des discours dont ils sont les supports. L'historien doit ainsi s'efforcer de gérer ces discours, à la fois lorsqu'il étudie les traces du passé et lorsqu'il tente d'en rendre compte en l'intellectualisant, ce qui est le propre du travail historique. Cet article considère ainsi les deux niveaux de confrontation des historiens à ce que Foucault nomme les discours.

\section{Mots-clefs}

Historiographie, hérésie, Moyen Âge, catharisme, discours.

\section{Abstract}

This work aims at giving a few grounds for thought as regards the latest historiography of Medieval dissidence. The issue is here a means to grasp the ressources historians can resort to define the two levels of reality : a clear distinction must indeed be made between the phenomena themselves and the discourses based on them. Historians must therefore attempt to manage these discourses, whether it is when reading traces from the past or when intellectualizing which is what historical writing is about. This article thus considers the two levels of confrontation between history and what Foucault calls the discourses.

\section{Keywords}

Historiography, heresy, Middle Ages, catharism, discourses. 